\title{
Innovations in CKRT: individualized therapy with fewer complications
}

\section{Balazs Szamosfalvi ${ }^{\bowtie}$ and Lenar Yessayan}

Continuous kidney replacement therapy (CKRT) can be a lifesaving intervention for critically ill patients; however, mortality remains high. The adaptation of existing innovations, including anti-clotting measures; cloud-computing for optimized treatment prescribing and therapy monitoring; and real-time sensing of blood and/or filter effluent composition to CKRT devices has the potential to enable personalized care and improve the safety and efficacy of this therapy.

The next 5 years could be an era of rapid progress

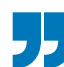

Department of Medicine, University of Michigan, Ann Arbor, MI, USA.

凶e-mail: bszamos@ med.umich.edu
Continuous kidney replacement therapy (CKRT) is used to treat critically ill patients in whom intermittent haemodialysis (IHD) or acute peritoneal dialysis is precluded. From its introduction in 1977 to the 1990s, a number of innovations took place, including the development of double-lumen central venous catheters; roller pumps to enable circulation of blood as well as fresh and waste CKRT fluids; sensors to detect fluid pressure and leakage of blood into waste fluid; the hollow fibre bundle artificial kidney; and scale-based balancing of fresh and waste CKRT fluids. These innovations culminated in the development of the first multi-purpose dedicated CKRT machine in the 1990s. The past 25 years have seen only minor improvements. However, a steady accumulation of clinical experience combined with innovations in IHD membranes, extra-corporeal organ support (ECOS) cartridges, sensors and cloud computing, set the stage for transformative changes in several areas.

\section{CKRT and ECOS integration}

The mortality of acute kidney injury (AKI) requiring CKRT in the intensive care unit (ICU) is high, at around $40 \%$, highlighting the need for novel therapeutic approaches. Flexible integration of CKRT circuits with various ECOS cartridges have potential to improve patient outcomes. However, despite the promise of ECOS cartridges in early studies, multi-centre randomized, controlled studies of some ECOS have shown a lack of benefit in critically ill patients. Such disappointing outcomes are perhaps due in part to practical issues with the timing and delivery of ECOS in the critical care setting. Filter sets such as the oXiris CKRT filter may facilitate the early removal of endotoxins and cytokines and improve outcomes for patients who require CKRT in the critical care setting ${ }^{1}$. This device has been available in Europe for years but has only recently been adopted in the USA in the context of the COVID-19 pandemic. The selective cytopheretic device (SCD) is another cartridge that aims to reduce cytokine levels and attenuate organ damage by changing the phenotype of granulocytes and monocytes.
However, a pivotal trial of the SCD in patients with septic shock ${ }^{2}$ was halted owing to technical difficulties with CKRT and regional citrate anticoagulation (RCA), underscoring the need for optimal integration of CKRT and RCA in future studies.

\section{CKRT anticoagulation}

In the absence of anticoagulation, blood clotting mechanisms are activated upon the contact of blood with the surface of the tubing and filter, leading to clotting and blood loss. One anticoagulation strategy involves the 2D modification of blood-contacting surfaces. For example, the inner surfaces of hollow fibres used for IHD have been modified by the binding of heparin ${ }^{1}$, the addition of a polyvinyl-pyrrolidone hydrogel layer, the use of the 'Lotus-effect' system, which has alternating hydrophobic and hydrophilic domains, or the covalent addition of surface-modifying macromolecules. However, surface-modified IHD filters cannot be inserted into most CKRT circuits, which are welded into a single unit comprising the blood tubing and filter. To enable use of these modified filters in CKRT systems, CKRT disposable sets that integrate advanced, coagulation-resistant filters should be made available for purchase, or open, filter-less CKRT circuits should be made available, to which providers can connect an IHD filter.

3D anticoagulants such as heparin or citrate-based solutions can be infused into CKRT circuits. However, heparin is of limited use owing to the risk of systemic bleeding. RCA can theoretically block CKRT circuit clotting without causing systemic bleeding, but its use has remained limited owing to severe complications that can arise. Of note, the combined use of highly optimized dialysers that remove $>80 \%$ of the infused citrate in a single pass during CKRT and coordinated citrate and calcium infusion pumps and a CKRT circuit optical haematocrit sensor to calculate the plasma clearance of citrate and calcium ${ }^{3}$ eliminated electrolyte complications and clotting in a large CKRT programme ${ }^{4}$. The addition of devices to optically sense CKRT waste fluid organic 
molecules ${ }^{5}$ and calcium ${ }^{6}$ combined with control software will enable full automation of RCA without the historic complications.

\section{Measurement of solute clearance}

About half of patients on CKRT receive antibiotics for life-threatening infections; any imbalance between CKRT removal and intravenous administration of an antibiotic can lead to underdosing or overdosing. Online clearance (OLC) measurements of small and medium size solute markers should be implemented during CKRT to precisely predict the removal of water-soluble, non-protein-bound antibiotics and enable personalized antibiotic dosing. Ionic dialysance (Kecn)-based OLC measurements of a commercial IHD machine have been clinically adapted for CKRT use ${ }^{4}$. Future CKRT devices should integrate (disposable) conductivity sensors and existing technology for online CKRT fluid generation to enable rapid modulation of the fresh CKRT fluid sodium level (which determines conductivity) for Kecn-based OLC measurements. UV adsorption-based OLC measurements such as those available on IHD machines ${ }^{7}$ could also be adapted to CKRT by, for example, adding a UV-absorbing marker molecule to the pre-filter citrate infusion. Finally, fluorescent markers used to measure glomerular filtration rate could be adapted for CKRT OLC measurements and detected by optical sensors on both the pre-filter and post-filter blood tubing and/or the waste fluid line ${ }^{8}$.

\section{CKRT for diagnostic use}

CKRT systems have the potential to be used diagnostically as continuous blood sampling devices. For example, incorporation of an existing IHD optical chamber and sensor into a CKRT circuit in combination with RCA enabled continuous monitoring of central venous haemoglobin level and oxygen saturation $\left(\mathrm{ScVO}_{2}\right)$ at minimal cost and without invasive procedural risk 9 Data on $\mathrm{ScVO}_{2}$ trends could help to identify relative changes in cardiac output. Analysis of the composition of the filter effluent will enable indirect monitoring of the solute composition of CKRT circuit blood and systemic blood $^{6}$. For example, spectroscopy approaches have been used to monitor lactate, glucose and urea levels in the dialyser effluent of IHD machines with clinically useful accuracy ${ }^{5}$. Changes in lactate level have been correlated with outcomes in the ICU, and glucose monitoring could potentially be used to detect systemic hypoglycaemia.

\section{Cloud-based computing services}

In the future, clinician-defined goals of CKRT could be translated into computationally optimized CKRT machine settings and fluid composition prescriptions using a progressive web application deployed on cloud servers, eliminating the impact of limited expertise and human error from CKRT prescribing. Data from the CKRT machine pumps and pressure, optical and conductivity sensors could be automatically collected into standardized cloud databases and made accessible to providers and manufacturers for near real-time CKRT programme quality assessment and improvement, and for monitoring of post-market outcomes.

\section{CKRT business model for innovation}

Currently, CKRT machines are produced in small numbers and cost two to four times as much as IHD machines. CKRT manufacturers have an ongoing revenue stream from the sale of custom CKRT blood circuits and sterile CKRT fluids in bags; disposables with limited shelf-life are manufactured in quantities just sufficient to meet their average use. To limit costs, hospitals typically purchase the minimum supplies needed. Return on investment calculations for manufacturers may not favour innovation. The shortage of CKRT machines and disposables encountered worldwide during the COVID-19 pandemic highlights the profound vulnerability of this approach. An alternative business model that might be more able to meet fluctuations in demand could be achieved by converging machine technology and blood circuits used for CKRT and home haemodialysis (HHD), and by using IHD filters for both. This approach will provide economies of scale and tap into the innovative developments that are happening for HHD. For example, volumetric blood pumps for single-needle access technology ${ }^{10}$ could be implemented first in the ICU and then transitioned to HHD. The 'Internet of Things' with cloud computing would allow the manufacturer to receive recurring payments for each hour a CKRT system is used in return for CKRT machine service, cloud-based data analytics and all disposables. This model would encourage the adoption of inexpensive online CKRT fluid generation using existing technologies from IHD and HHD with the benefit of enabling easy personalization of CKRT fluid and the logistical advantage of unlimited access to online CKRT fluid and IHD filters in times of increased utilization.

CKRT machines have seen only modest changes in recent years. The next 5 years could be an era of rapid progress as most innovations that we need to make CKRT easier and safer to use as part of broader multi-organ support in the ICU are already available. Enlightened stewardship by key medical opinion leaders and a commitment to producing results by all stakeholders will be needed for the success of this journey.

1. Broman, M. E. et al. Endotoxin and cytokine reducing properties of the oXiris membrane in patients with septic shock: a randomized crossover double-blind study. PLOS ONE 14, e0220444 (2019).

2. Tumlin, J. A. et al. A multi-center, randomized, controlled, pivotal study to assess the safety and efficacy of a selective cytopheretic device in patients with acute kidney injury. PLOS ONE 10, e0132482 (2015)

3. Szamosfalvi, B., Frinak, S. \& Yee, J. Automated regional citrate anticoagulation: technological barriers and possible solutions. Blood Purif. 29, 204-209 (2010).

4. Szamosfalvi, B., Frinak, S. \& Yee, J. Sensors and hybrid therapies: a new approach with automated citrate anticoagulation. Blood Purif 34, 80-87 (2012).

5. Roth, A. et al. Infrared spectroscopy in hemodialysis: reagent-free monitoring of patient detoxification by infrared spectroscopy. Anal. Bioanal. Chem. 403, 391-399 (2012).

6. Sharma, M. K. et al. On-line monitoring of electrolytes in hemodialysis: on the road towards individualizing treatment. Expert Rev. Med. Devices 13, 933-943 (2016).

7. Daugirdas, J. T. \& Tattersall, J. E. Automated monitoring of hemodialysis adequacy by dialysis machines: potential benefits to patients and cost savings. Kidney Int. 78, 833-835 (2010). 8. Shieh, J. J. et al. Characterization of MB-102, a new fluorescent tracer agent for point-of-care renal function monitoring. J. Pharm. Sci. 109, 1191-1198 (2020).

9. Yessayan, L., Friank, S. \& Szamosfalvi, B. Online hemoglobin and oxygen saturation sensing during continuous renal replacement therapy with regional citrate anticoagulation. ASAIO J. 61, 489-495 (2015).

10. Shu, F. et al. Multimodal flow visualization and optimization of pneumatic blood pump for sorbent hemodialysis system. Artif. Organs 33, 334-345 (2009).

\section{Competing interests}

The authors declare no competing interests. 